Research Article

\title{
Metastatin as a Marker for Hyperandrogenemia in Iraqi Women with Polycystic Ovary Syndrome
}

\author{
Manal Madany Abdalqader $(\mathbb{D}$ and Shatha Sami Hussein \\ College of Medicine, Department of Obstetrics \&Gynecology, Al-Mustansiriyah University, Baghdad, Iraq \\ Correspondence should be addressed to Manal Madany Abdalqader; manalmadany@uomustansiriyah.edu.iq
}

Received 30 March 2020; Revised 3 August 2020; Accepted 13 September 2020; Published 25 September 2020

Academic Editor: Diego Raimondo

Copyright (c) 2020 Manal Madany Abdalqader and Shatha Sami Hussein. This is an open access article distributed under the Creative Commons Attribution License, which permits unrestricted use, distribution, and reproduction in any medium, provided the original work is properly cited.

Objective. Kisspeptin 1 might reflect increased androgen level in polycystic ovarian syndrome instead of other markers. Study Design. A case control study was performed in Al-Yarmouk Teaching Hospital from $1^{\text {st }}$ of July 2016 to $1^{\text {st }}$ of July 2017; it involved 87 women divided into two groups: 44 women diagnosed as PCOS, 22 women with BMI $\geq 25 \mathrm{~kg} / \mathrm{m}^{2}$ and 22 women with $\mathrm{BMI}<25 \mathrm{~kg} / \mathrm{m}^{2}$, and another 43 women without PCOS, 22 women with BMI $\geq 25 \mathrm{~kg} / \mathrm{m}^{2}$ and 21 women with BMI $<25 \mathrm{~kg} / \mathrm{m}^{2}$. Hormonal, metabolic profiles, and hirsutism scores, as well as serum kisspeptin level, were assessed by using Human Kisspeptin 1(KISS-1) ELISA Kit. The blood samples between days 2 and 5 of menstrual cycle were drawn by an disposable sterile syringe and collected in EDTA containing tubes (as anticoagulant), and the hormonal profile was measured using a biotech ELISA reader. Result. Serum level of kisspeptin was significantly higher in PCOS compared to control (322.4 vs. $235.3 \mathrm{ng} / \mathrm{L}$, respectively). There was no significant difference in age, BMI, and parity between control and PCOS; the frequency of hirsutism, acne, elevated LH, and increased free testosterone (fTT) were significantly higher in PCOS compared to control. Kisspeptin shows a direct significant correlation with hirsutism and fTT $(r=0.648,0.238$, respectively). In ROC analysis, kisspeptin had AUC (95\% CI) $=0.874$ (0.785-0.935) for predicting PCOS. Conclusion. Kisspeptin levels might be used as a marker for hyperandrogenemia in polycystic ovarian syndrome.

\section{Introduction}

Polycystic ovarian syndrome (PCOS) is regarded as one of the commonest endocrinopathic disorders in women at the reproductive age group with a prevalence of about $6-12 \%$ [1]. It is usually characterized by multiple hormonal imbalances, reflecting as a sign and symptom of hyperandrogenism, which leads to multiple health effects on females in the form of early and late sequelae [2], with androgen excess, oligomenorrhea or an ovulation, and ultrasound finding of polycystic ovary appearance. In addition, other abnormalities such as insulin resistance, inflammation, obesity, cardiovascular changes, and infertility are common among women with PCOS [3]. Although up to $70 \%$ of women with PCOS may be undiagnosed and due to high prevalence of PCOS [4], all cases of hirsutism during puberty or in women of reproductive age group merit investigations for sex hormone level, pelvic ultrasonography, to exclude this syndrome [5].

A pivotal role for specific proinflammatory mediators in the pathogenesis of PCOS was described in recent evidence which elucidated a new pathway on the etiological considerations for PCOS, as it recently considered a chronic, low-grade inflammatory abnormality, independent for the presence of obesity [6]. Kisspeptin is a gene product, which usually resulted from an initial 145-amino acid peptide which cleaved to a 54-amino acid protein [7]. Kisspeptin has a role in gonadotropin-releasing hormone $(\mathrm{GnRH})$ neuron activation in the hypothalamus, and as a result, it causes GnRH release which leads to FSH and LH release. Kisspeptin/GPR54 plays a major role in the initial sexual development which is found in mice and sexually immature humans with mutations that block the expression of the GPR54 gene [8]. 
Several independent lab groups support the role of kisspeptin in the hypogonadotropic hypogonadism pathway. GPR54 mutation was found to be the reason for this abnormality, as it was noted that people who had this mutation, or missing GPR54, have abnormality in the development of gonads at puberty. Other form of phenotypes was connected to this mutation including gonadotropin concentrations, a low level of sex steroid in blood, and sterility which led to discover the role of Kisspeptin in initiation of puberty by triggering the neurons which participate in GnRH release and the possible role on of luteinizing hormone (LH) and follicle-stimulating hormone (FSH) release [9]. Efforts were made to understand the regulatory mechanism of kisspeptin and its gene expression and more exactly detect the mechanism behind its role in $\mathrm{GnRH}$ and $\mathrm{LH}$ release.

Because of the complicated relation seen between kisspeptin and the hypothalamic-pituitary-gonadal axis, the current work planned to measure the level of kisspeptin in polycystic ovary syndrome (PCOS) and analyze the relation between kisspeptin and PCOS-related reproductive and associated metabolic changes.

\section{Materials and Methods}

2.1. Study Design. A case control study was carried out in Obstetric and Gynecological Department at Al-Yarmouk Teaching Hospital through the period from $1^{\text {st }}$ of July 2016 to $1^{\text {st }}$ of July 2017. A total of 87 women participated in this study and were divided into 2 groups: group A: 44 women diagnosed as PCOS (divided into 22 women with $\mathrm{BMI} \geq 25 \mathrm{~kg} / \mathrm{m}^{2}$ and 22 women with $\mathrm{BMI}<25 \mathrm{~kg} / \mathrm{m}^{2}$ ) and group B: 43 women without PCOS (divided into 22 women with $\mathrm{BMI} \geq 25 \mathrm{~kg} / \mathrm{m}^{2}$ and 21 women with $\mathrm{BMI}<25 \mathrm{~kg} / \mathrm{m}^{2}$ ).

2.2. Inclusion and Exclusion Criteria. Inclusion criteria included women diagnosed as PCOS according to the Rotterdam criteria [10] in the form of oligomenorrhea \pm an ovulation, ultrasound of polycystic ovaries (follicular cyst number $\geq 12$ ), and biochemical and/or clinical evidence of hyperandrogenism. Exclusion criteria included any cause of hyperandrogenism other than PCOS as congenital adrenal hyperplasia, virilization tumors, Cushing syndrome, prolactinoma, hypertension, diabetes, and cardiovascular disorders.

2.3. Data Collection. Full history regarding signs and symptoms (hirsutism, acne, menstrual irregularity, amenorrhea etc.) and examination which includes BMI, hirsutism evaluation according to modified Ferriman-Gallwey score $\geq 8$ [11] was collected; calculation of their BMI included the ratio of weight divided by the square of height $\left(\mathrm{kg} / \mathrm{m}^{2}\right)$. Routine laboratory investigation was performed including fasting plasma glucose (FPG), HDL, LDL, triglyceride, LH, $\mathrm{FSH}$, TSH, sex hormone-binding globulin (SHBG), dehydroepiandrosterone sulfate (DHEAS), and testosterone level which were all taken at the morning after sample for serum overnight fasting at the early follicular phase $\left(2^{\text {nd }}-5^{\text {th }}\right.$ day of the menstrual cycle). Kisspeptin levels were also taken and performed by an enzyme immunoassay kit. Based on the findings of Gorkem et al. [12] with mean difference of mean (1.11) and standard deviation (SD) for PCOS (2.11) and SD for control (2.16) for kisspeptin, with type I error $(\alpha)=0.10$ and type II error $(\beta)=80 \%$, the sample size was estimated to be 47 for PCOS and 47 for control.

2.4. Flowchart. After initial selection of the groups, $8.5 \%$ (4 women) of subjects in PCOS and $6.3 \%$ (3 women) in the control group were excluded from the study. Since both were less than $20 \%$ (which is considered significant bias), the authors did not consider this lost to follow-up because it would create significant bias [13].

2.5. Statistical Analysis. The age was presented as mean and standard deviation as it was normally distributed, while both parity and kisspeptin were presented as median and interquartile range (did not follow normal Mann distribution, assessed by Kolmogorov-Smirnov test for continuous data distribution). Whitney $U$ nonparametric test (twotailed) was used to compare the continuous variables; receiver operating characteristic (ROC) curve was used to assess the sensitivity between study groups. Pearson's chisquare test was used to assess specificity of kisspeptin of participants in both groups and to assess statistical association between the categorical parameter levels and the study groups. Linear regression analysis was used to assess the relationship between kisspeptin and other parameters. ROC analysis was performed to assess the diagnostic validity of kisspeptin for PCOS. A $p$ value less than 0.05 was significant, and all the statistical analyses were performed using SPSS 22.0.0 (Chicago, IL) and MedCalc Statistical Software version 14.8.1 (MedCalc Software bvba, Ostend, Belgium; 2014) (Figure 1).

2.6. Results. There was no significant difference in age, BMI, and parity between control and PCOS; the frequency of hirsutism, acne, elevated LH, and increased free testosterone (fTT) were significantly higher in PCOS compared to control, and serum levels of kisspeptin were also significantly higher in PCOS (this maintained even after dividing both groups according to their BMI, see Figure 2), as illustrated in Table 1.

Kisspeptin shows a direct significant correlation with hirsutism and fTT as illustrated in Table 2.

Kisspeptin shows good ability (since AUC between 0.8 and 0.89 ) to predict PCOS, since SP (specificity) is higher than SN (sensitivity), and also PPV (positive predictive value) is higher than NPV (negative predictive value); this suggests it may be a more useful tool for confirmation of the diagnosis of PCOS, as illustrated in Table 3 and Figure 3.

In multivariate analysis, kisspeptin shows an independent relationship with PCOS (after excluding the effect possible confounders in this study such as age, BMI, LH, and fTT) in which an increase in $1 \mathrm{ng} / \mathrm{L}$ of kisspeptin increased the probability of PCOS by $3.9 \%$ (ranging from $1.9 \%$ to $5.9 \%$; ARR: 1.039-1.0), as illustrated in Table 4. 


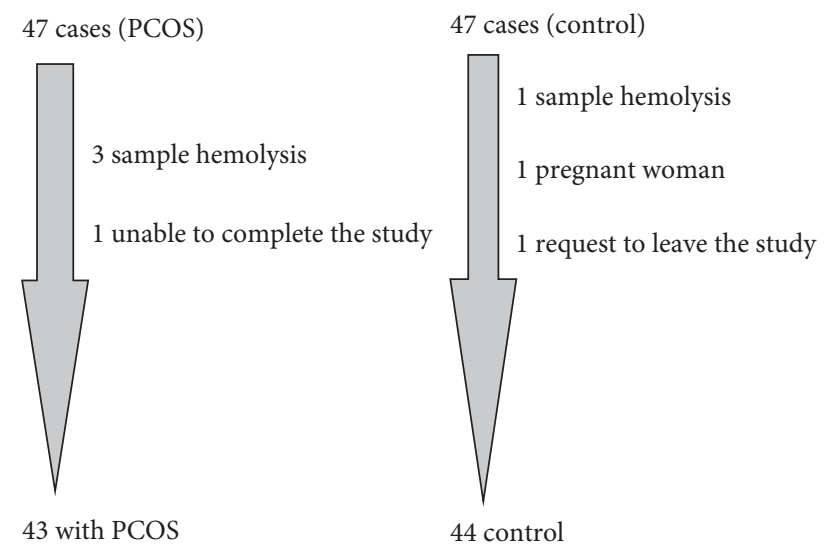

FIgURE 1: Flowchart of the study.

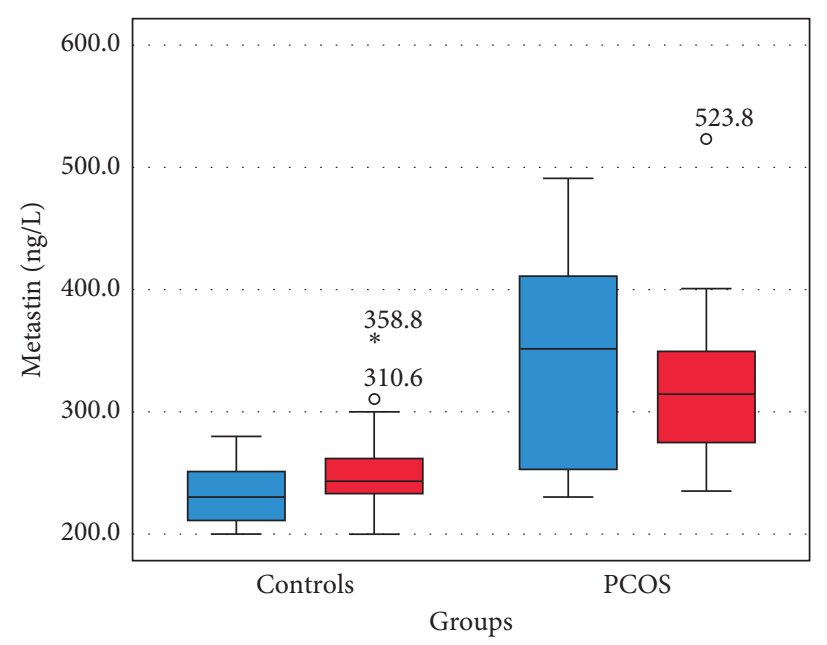

BMI

$\square<25 \mathrm{~kg} / \mathrm{m}^{2}$

$\geq 25 \mathrm{~kg} / \mathrm{m}^{2}$

Figure 2: Plasma kisspeptin (metastin) levels of women with regard to the body mass index (asterisk and small circles represent the outliers).

TABLE 1: Comparison between control and PCOS women.

\begin{tabular}{|c|c|c|c|}
\hline Parameters & Control & PCOS & $p$ value \\
\hline Number & 44 & 43 & - \\
\hline Age (years), mean $\pm S D$ & $24.5 \pm 5.6$ & $26.3 \pm 6.0$ & 0.162 \\
\hline Parity, median (IQR) & $1(0.25-2.0)$ & $2(1-3)$ & 0.120 \\
\hline Kisspeptin (ng/L), median (IQR) & $235.3(220.6-258.4)$ & $322.4(254.6-377.1)$ & $<0.001$ \\
\hline \multicolumn{4}{|l|}{ BMI, $n(\%)$} \\
\hline$<25 \mathrm{~kg} / \mathrm{m}^{2}$ & $21(47.7 \%)$ & $23(53.5 \%)$ & \multirow{2}{*}{0.591} \\
\hline$\geq 25 \mathrm{~kg} / \mathrm{m}^{2}$ & $23(52.3 \%)$ & $20(46.5 \%)$ & \\
\hline Hirsutism, $n(\%)$ & $0(0 \%)$ & $43(100 \%)$ & $<0.001$ \\
\hline Acne, $n(\%)$ & $18(40.9 \%)$ & $27(62.8 \%)$ & 0.041 \\
\hline Elevated LH, $n(\%)$ & $14(31.8 \%)$ & $27(62.8 \%)$ & 0.004 \\
\hline \multicolumn{4}{|l|}{$\mathrm{FSH}, n(\%)$} \\
\hline Normal & $31(70.5 \%)$ & $28(65.1 \%)$ & \multirow{3}{*}{0.700} \\
\hline Decreased & $9(20.5 \%)$ & $12(27.9 \%)$ & \\
\hline Elevated & $4(9.1 \%)$ & $3(7 \%)$ & \\
\hline Increased TSH, $n(\%)$ & $11(25 \%)$ & $13(30.2 \%)$ & 0.585 \\
\hline Increased PRL, $n(\%)$ & $20(45.5 \%)$ & $15(34.9 \%)$ & 0.315 \\
\hline SHBG, $n(\%)$ & & & \\
\hline
\end{tabular}


TABLE 1: Continued.

\begin{tabular}{|c|c|c|c|}
\hline Parameters & Control & PCOS & $p$ value \\
\hline Normal & $31(70.5 \%)$ & $29(67.4 \%)$ & \\
\hline Decreased & $11(25 \%)$ & $12(27.9 \%)$ & 0.952 \\
\hline Elevated & $2(4.5 \%)$ & $2(4.7 \%)$ & \\
\hline Increased testosterone, $n(\%)$ & $18(40.9 \%)$ & $28(65.1 \%)$ & 0.024 \\
\hline \multicolumn{4}{|l|}{ DHEAS, $n(\%)$} \\
\hline Normal & $31(70.5 \%)$ & $30(69.8 \%)$ & \\
\hline Decreased & $8(18.2 \%)$ & $7(16.3 \%)$ & 0.923 \\
\hline Elevated & $5(11.4 \%)$ & $6(14 \%)$ & \\
\hline \multicolumn{4}{|l|}{ Glycemic status, $n(\%)$} \\
\hline Normal & $25(56.8 \%)$ & $21(48.8 \%)$ & \\
\hline Decreased & $9(20.5 \%)$ & $9(20.9 \%)$ & 0.695 \\
\hline Elevated & $10(22.7 \%)$ & $13(30.2 \%)$ & \\
\hline Impaired lipid profile & $14(31.8 \%)$ & $16(37.2 \%)$ & 0.597 \\
\hline
\end{tabular}

SD: standard deviation, $n$ : number, IQR: interquartile range $\left(25^{\text {th }}-57^{\text {th }}\right.$ percentile), PRL: prolactin, SHBG: sex hormone-binding globulin, T: testosterone, DHEAS: dehydroepiandrosterone sulfate, and FPG: fasting blood glucose.

TABLE 2: Correlations between kisspeptin with demographic and clinical characteristics and hormonal and metabolic profiles of all subjects (including controls).

\begin{tabular}{lcc}
\hline Parameters & Correlation coefficient $(r)$ & $p$ value \\
\hline Age & 0.016 & 0.886 \\
Parity & 0.131 & 0.228 \\
BMI & 0.066 & 0.541 \\
Hirsutism & 0.648 & $<0.001^{*}$ \\
Acne & 0.073 & 0.500 \\
LH & 0.139 & 0.199 \\
FSH & -0.194 & 0.072 \\
TSH & -0.045 & 0.679 \\
PRL & -0.133 \\
SHBG & -0.032 & 0.218 \\
Testosterone & 0.238 & 0.772 \\
DHEAS & -0.101 & $0.027^{*}$ \\
FPG & -0.064 & 0.353 \\
\hline
\end{tabular}

PRL: prolactin, SHBG: sex hormone-binding globulin, DHEAS: dehydroepiandrosterone sulfate, and FPG: fasting blood glucose.

TABLE 3: ROC analysis of the validity of kisspeptin as a predictor of PCOS.

\begin{tabular}{lcccccccc}
\hline ROC $(95 \%$ CI $)$ & Cutoff & SN & SP & AC (\%) & PPV & NPV & + LH & - LH \\
\hline $0.874(0.785-0.935)$ & $\geq 271.234$ & 74.4 & 88.6 & 81.6 & 86.5 & 78.0 & 6.55 & 0.29 \\
\hline
\end{tabular}

SN: sensitivity, SP: specificity, AC: accuracy, PPV: positive predictive value, NPV: negative predictive value, and LH: likelihood ratio.

\section{Discussion}

PCOS is related to a disturbance in the hypothalamic-pituitarygonadal axis [14]. Kisspeptin has been recognized by its important role in $\mathrm{GnRH}$ secretion initiation at puberty. Kisspeptin has been recognized by its important role in $\mathrm{GnRH}$ secretion initiation at puberty, regulatory secretion of $\mathrm{LH}$ during ovulation process, and its relation to PCOS was found to be implicated in the hypothalamus-pituitary-ovary axis disturbance observed in polycystic ovary syndrome (PCOS) [15].

The current study showed that kisspeptin was significantly higher in PCOS compared to control (322.4 vs. $235.3 \mathrm{ng} / \mathrm{L}$ ), and after performing ROC analysis, kisspeptin showed good ability to predict PCOS, with serum level $\geq 271.234 \mathrm{ng} / \mathrm{L}$ predicting PCOS; at this point, kisspeptin showed higher specificity (88.6\%) and lower sensitivity (74.4\%) with $81.6 \%$ accuracy. In addition, the positive likelihood ratio $(\mathrm{LH})$ was 6.55 which indicates that this test can increase the index of suspicion for confirming the PCOS diagnosis by 35-40\%, while its negative LH (0.29) indicates it has $25-30 \%$ ability to exclude the diagnosis of PCOS; this was in agreement with a recent Turkish study in 2018 with kisspeptin levels in a normal ovarian reserve which were $4.65 \pm 2.16 \mathrm{ng} / \mathrm{mL}$, and $5.76 \pm 2.11$ in those with a high ovarian reserve [12]. Jeon et al. showed similar findings $(10.65 \pm 6.14$ vs. $6.51 \pm 3.13 \mathrm{pmol} / 1, p<0.001)$ [16] and were in agreement with Umayal et al. (4.713 vs. $4.127 \mathrm{nmol} / \mathrm{L}, p$ value $=0.033$ ) [17]. Other studies such as Chen et al. and Joen et al. both showed increased kisspeptin level in women with PCOS $[16,18]$. Others showed no significant difference 


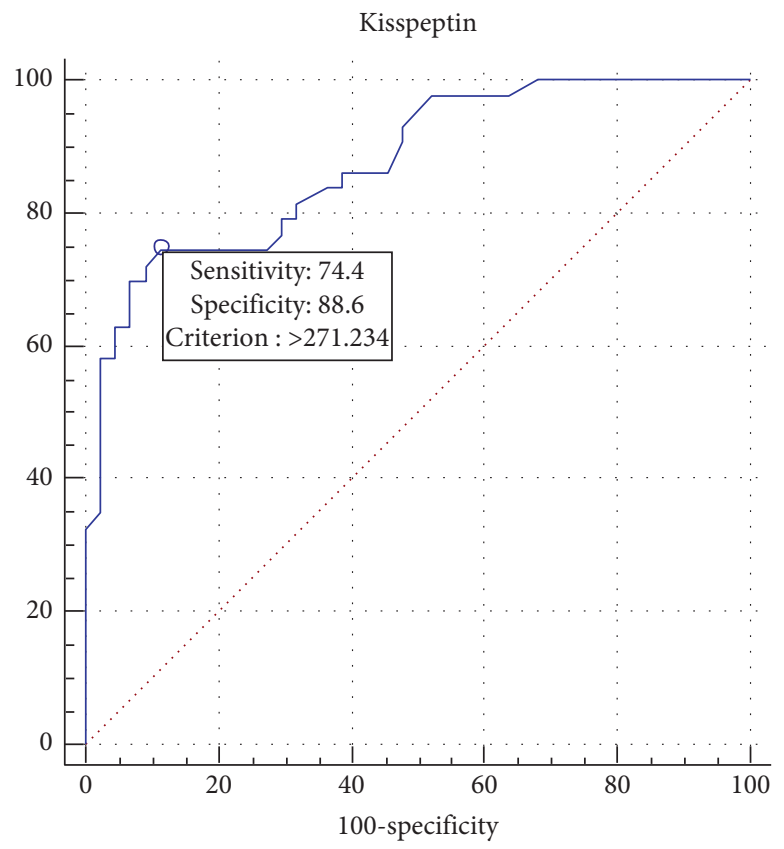

Figure 3: ROC analysis for kisspeptin levels between the PCOS and the control groups.

TABLE 4: Multiple regression analysis for the $e$ factors that affect PCOS.

\begin{tabular}{lccc}
\hline Parameters & OR $(95 \% \mathrm{CI})$ & Wald & $p$ value \\
\hline Age & $1.106(0.987-1.240)$ & 2.999 & 0.083 \\
BMI & $0.440(0.110-1.755)$ & 1.353 & 0.245 \\
Kisspeptin & $1.039(1.019-1.059)$ & 15.313 & $>0.001[\mathrm{~S}]$ \\
LH & $12.002(2.543-56.651)$ & 9.852 & $0.002[S]$ \\
Free TT & $3.385(0.870-3.179)$ & 3.092 & 0.079 \\
\hline
\end{tabular}

$R^{2}($ Cox and Snell $)=0.514$.

between PCOS and control, such as Ozay et al. [19] with kisspeptin levels $1.92 \pm 1.29$ vs. $1.49 \pm 1.46 ; p$ value $=0.638$ [19], all these indicate that kisspeptin had good potential as a marker for PCOS diagnosis.

Some studies showed that kisspeptins may play a role in the regulation of islet function by stimulating insulin secretion from mouse and human islets. Adipose tissue acts as an endocrine organ, and its function may be regulated by kisspeptins [20]. It has been shown that peptides have more gonadotropin secreted through the activation of the central hypothalamic-pituitary-gonadal (HPG) axis at the hypothalamic level, with the gonadotropin stimulatory effects of kisspeptin being abolished by preadministration of a $\mathrm{GnRH}$ antagonist [21]; therefore, kisspeptin was found to have a regulatory effect through the activation of $\mathrm{GnRH}$ neurons, making it the first step for the last common pathway associated with controlling reproduction.

Increased HPG axis stimulation may be associated with reproductive function restoration for PCOS women, while overstimulation of this pathway axis produces downregulation of reproductive hormone secretion, and KISS-1 is found to have a role in treating prostate and breast malignancies, as well as benign hormone-dependent disorders as benign prostate hyperplasia and endometriosis, through its act as a suppresser in the metastatic cascade [22].

\section{Conclusion}

In the current study, the effects of kisspeptin on the release of reproductive hormones were examined. All the above findings have identified kisspeptin as a possible diagnostic marker for PCOS.

\section{Data Availability}

The patient data used to support the findings of this study are currently under embargo, while the research findings are commercialized. Requests for data 6/12 months after publication of this article will be considered by the corresponding author.

\section{Ethical Approval}

This study was approved by the Institutional Review Board of the local hospital (Al-Yarmouk Teaching Hospital), in accordance with the Declaration of Helsinki 2013; in 
addition, participated women received appropriate management by the researchers.

\section{Consent}

Written informed consent was obtained from each woman before participating in the study.

\section{Conflicts of Interest}

The authors declare that they have no conflicts of interest.

\section{Acknowledgments}

The authors would like to express their deepest appreciation to all those who provided them the possibility to complete this article.

\section{References}

[1] P. Wojciechowski, A. Lipowska, P. Rys et al., "Impact of FTO genotypes on BMI and weight in polycystic ovary syndrome: a systematic review and meta-analysis," Diabetologia, vol. 55, no. 10, pp. 2636-2645, 2012.

[2] K. Farrell and M. H. Antoni, "Insulin resistance, obesity, inflammation, and depression in polycystic ovary syndrome: biobehavioral mechanisms and interventions," Fertility and Sterility, vol. 94, no. 5, pp. 1565-1574, 2010.

[3] H. Teede, A. Deeks, and L. Moran, "Polycystic ovary syndrome: a complex condition with psychological, reproductive and metabolic manifestations that impacts on health across the lifespan," BMC Medicine, vol. 8, no. 1, p. 41, 2010.

[4] J. Boyle and H. J. Teede, "Polycystic ovary syndrome-an update," Australian Family Physician, vol. 41, no. 10, pp. 752-756, 2012.

[5] R. Azziz, E. Carmina, D. Dewailly et al., "The androgen excess and PCOS society criteria for the polycystic ovary syndrome: the complete task force report," Fertility and Sterility, vol. 91, no. 2, pp. 456-488, 2009.

[6] H. F. Escobar-Morreale, M. Luque-Ramírez, and F. González, "Circulating inflammatory markers in polycystic ovary syndrome: a systematic review and metaanalysis," Fertility and Sterility, vol. 95, no. 3, pp. 1048-1058, 2011.

[7] E. J. Mead, J. J. Maguire, R. E. Kuc, and A. P. Davenport, "Kisspeptins: a multifunctional peptide system with a role in reproduction, cancer and the cardiovascular system," British Journal of Pharmacology, vol. 151, no. 8, pp. 1143-1153, 2007.

[8] R. Young-Jun, "Kisspeptin/G protein-coupled receptor-54 system as an essential gatekeeper of pubertal development," Annals of Pediatric Endocrinology \& Metabolism, vol. 18, no. 2, 55 pages, 2013.

[9] J. Pasquier, N. Kamech, A.-G. Lafont, H. Vaudry, K. Rousseau, and S. Dufour, "Molecular evolution of GPCRs: kisspeptin/kisspeptin receptors," Journal of Molecular Endocrinology, vol. 52, no. 3, pp. T101-T117, 2014.

[10] B. Fauser, "Revised 2003 consensus on diagnostic criteria and long-term health risks related to polycystic ovary syndrome," Fertility and Sterility, vol. 81, no. 1, pp. 19-25, 2004.

[11] R. Aswini and S. Jayapalan, "Modified ferriman-gallwey score in hirsutism and its association with metabolic syndrome," International Journal of Trichology, vol. 9, no. 1, 7 pages, 2017.

[12] U. Gorkem, C. Togrul, E. Arslan, A. Sargin Oruc, and N. Buyukkayaci Duman, "Is there a role for kisspeptin in pathogenesis of polycystic ovary syndrome?" Gynecological Endocrinology, vol. 34, no. 2, pp. 157-160, 2018.

[13] J. Dettori, "Loss to follow-up," Evidence-Based Spine-Care Journal, vol. 2, no. 1, pp. 7-10, 2011.

[14] S. F. Witchel and M. Tena-Sempere, "The kiss1 system and polycystic ovary syndrome: lessons from physiology and putative pathophysiologic implications," Fertility and Sterility, vol. 100, no. 1, pp. 12-22, 2013.

[15] M. Kondo, S. Osuka, A. Iwase et al., "Increase of kisspeptinpositive cells in the hypothalamus of a rat model of polycystic ovary syndrome," Metabolic Brain Disease, vol. 31, no. 3, pp. 673-681, 2016.

[16] Y. E. Jeon, K. E. Lee, J. A. Jung et al., "Kisspeptin, leptin, and retinol-binding protein 4 in women with polycystic ovary syndrome," Gynecologic and Obstetric Investigation, vol. 75, no. 4, pp. 268-274, 2013.

[17] B. Umayal, S. N. Jayakody, N. V. Chandrasekharan, W. S. Wijesundera, and C. N. Wijeyaratne, "Polycystic ovary syndrome (PCOS) and kisspeptin-a Sri Lankan study," Journal of Postgraduate Medicine, vol. 65, no. 1, pp. 18-23, 2019.

[18] X. Chen, Y. Mo, L. Li, Y. Chen, Y. Li, and D. Yang, "Increased plasma metastin levels in adolescent women with polycystic ovary syndrome," European Journal of Obstetrics \& Gynecology and Reproductive Biology, vol. 149, no. 1, pp. 72-76, 2010.

[19] O. E. Ozay, A. C. Ozay, B. Acar, E. Cagliyan, M. Seçil, and T. Küme, "Role of kisspeptin in polycystic ovary syndrome (PCOS)," Gynecological Endocrinology, vol. 32, no. 9, pp. 718-722, 2016.

[20] E. Yerlikaya, F. Akin, S. Turgut et al., "Relation of plasma kisspeptin levels with obesity and insulin resistance in polycystic ovary syndrome," Endocrine Abstracts, vol. 32, p. P619, 2013.

[21] C. N. Jayasena, G. M. K. Nijher, O. B. Chaudhri et al., "Subcutaneous injection of kisspeptin-54 acutely stimulates gonadotropin secretion in women with hypothalamic amenorrhea, but chronic administration causes tachyphylaxis," The Journal of Clinical Endocrinology \& Metabolism, vol. 94, no. 11, pp. 4315-4323, 2009.

[22] K. Skorupskaite, J. T. George, and R. A. Anderson, "The kisspeptin-GnRH pathway in human reproductive health and disease," Human Reproduction Update, vol. 20, no. 4, pp. $485-500,2014$. 